\title{
Synthesis and Characterization of Isolated Platinum Nanocrystals
}

\author{
T. Tyler , ${ }^{*}$ D. Elswick, $* *$ and J. Hren** \\ * International Technology Center, 8100-120 Brownleigh Drive, Raleigh, North Carolina 27617 \\ ** Department of Materials Science and Engineering, NCSU, Raleigh, N.C. 27695-7907
}

There is much interest in nanoscale materials due to their size-dependent properties, including variations in electronic bandgap, photoluminescence, and catalytic activity [1-4]. Platinum nanoparticles are of particular interest due in large part to their catalytic activity and potential applications in fuel cells. It has been shown that several factors affect the behavior of platinum nanoparticles as catalysts, including synthesis technique, size, and substrate material [5-7]. A means to isolate individual nanoparticles for characterization is therefore desirable. In this paper a technique for synthesis of isolated platinum nanocrystals is reported. The substrates used in this study (ultra-sharp aluminum needles) have the advantage of requiring no further sample preparation for observation in a transmission electron microscope. Preliminary characterizations were conducted, including x-ray energy-dispersive spectroscopy (EDS), transmission electron microscopy (TEM), and electron energy loss spectrometry (EELS).

Electrochemically etched aluminum needles were employed as substrates for the platinum nanocrystal synthesis. The etching process of the aluminum is described briefly: $99.999 \%$ aluminum wire (150 $\mu \mathrm{m}$ diameter) was biased (15-20 VAC) in a $20 \%$ perchloric acid (in methanol) solution at $-30^{\circ} \mathrm{C}$; the wire was etched until breaking, resulting in a pointed needle with a radius of curvature $\sim 50 \mathrm{~nm}$. The electrode used in the acid bath was a platinum wire ( $250 \mu \mathrm{m}$ diameter, $99.99 \%)$, which served as a source for platinum deposition onto the aluminum needle. Subsequent to etching and after undergoing field electron emission (experiments for which the sample was originally intended), it was discovered that the aluminum needle contained platinum nanocrystals on the surface (Figures 1 and 2). A mechanism for platinum deposition is proposed, which consists of: 1) the dissolution of the platinum electrode in solution, forming the

$\mathrm{PtCl}_{6}{ }^{2-}$ complex and 2) the plating out of the $\mathrm{Pt}^{4+}$ ion onto the aluminum during the negative halfcycle of the AC etching process. Field emission is thought to play a crucial role in nanocrystal formation; the high electric fields $\left(\sim 10^{7} \mathrm{~V} / \mathrm{cm}\right)$ and current densities $\left(\sim 10^{6} \mathrm{~A} / \mathrm{cm}^{2}\right)$ are believed to facilitate surface migration and crystallization of the plated platinum. To support the proposed mechanism, a set of samples was etched with varying conditions: AC, pulsed DC (half-wave rectified $\mathrm{AC}$ ), and $\mathrm{AC}$ followed by pulsed $\mathrm{DC}$, and then analyzed using x-ray energy dispersive spectroscopy; the results are summarized in Table 1. Further studies are underway to better understand this phenomenon.

\section{References}

[1] Buffat, D. A.; Borel, J. P. Phys. ReV. 1976, A13, 2289.

[2] Wilcoxon, J. P. et al., J. Chem. Phys. 1998, 108, 9137.

[3] Haruta, M., et al., J. Catal. 1993, 144, 175.

[4] Sakurai, H.; Haruta, M. Appl. Catal. A: General 1995, 127, 93.

[5] M. Watanabe, S. Saegusa, P. Stonehart, J. Electroanal. Chem. 271 (1989) 213.

[6] S. Katsuaki, I. Ryuhei, K. Hideaki, J. Electroanal. Chem. 284 (1990) 523.

[7] M.-S. L"offler, et al., J. Divisek, Phys. Chem. Chem. Phys. 3 (2001) 333. 

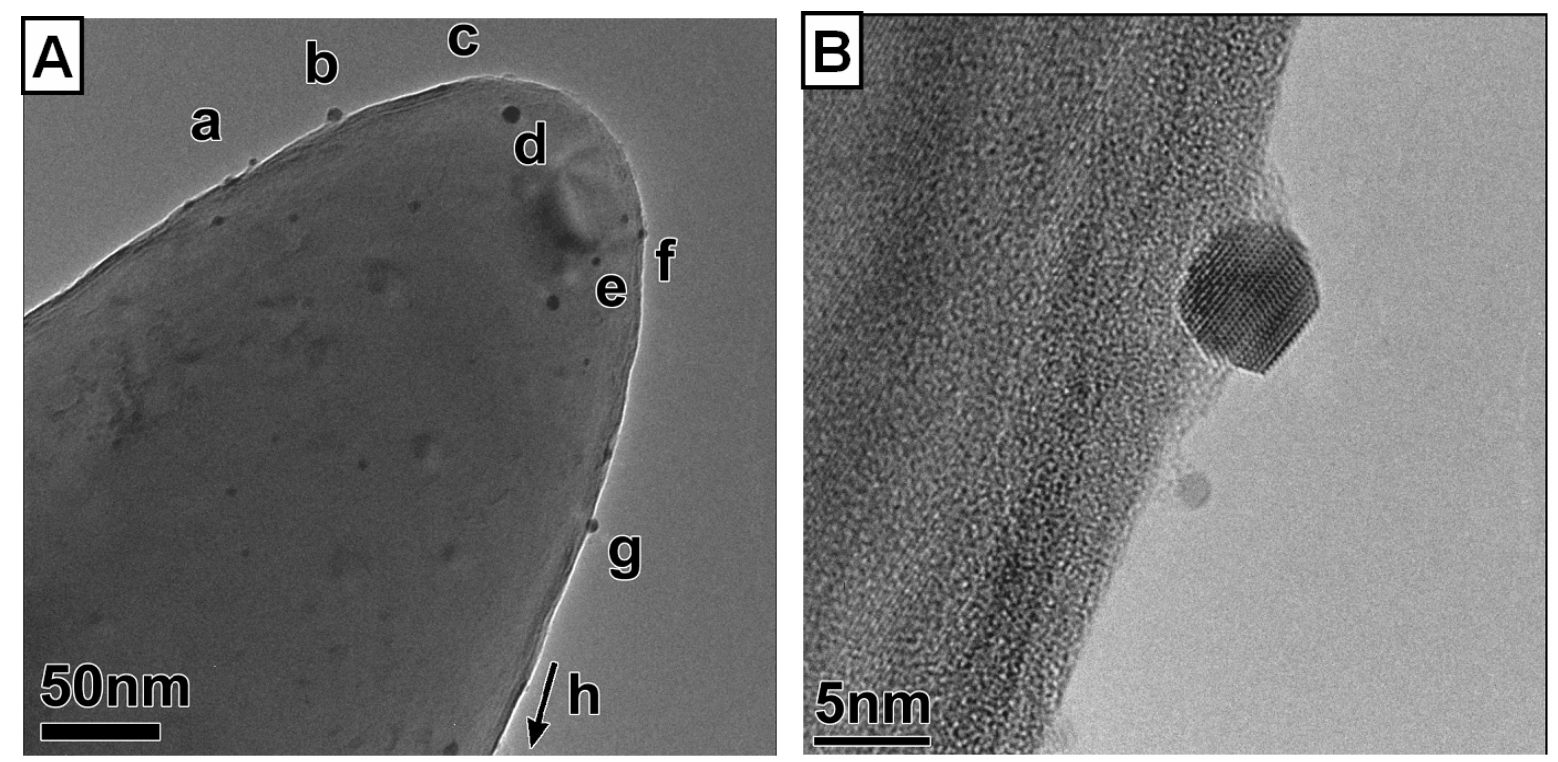

Fig. 1 A. Transmission electron micrograph of aluminum needle; labels indicate individual platinum nanoparticles. B. High magnification of platinum nanoparticle labeled "g."
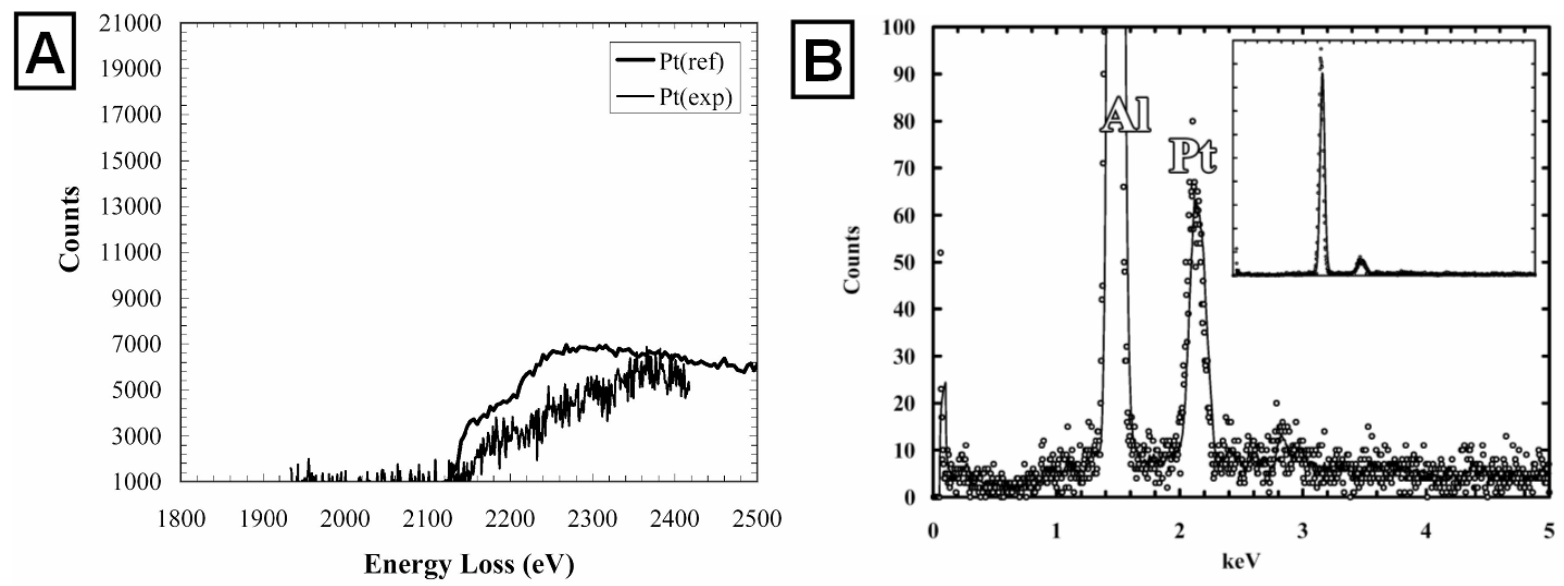

Fig. 2 A. Electron energy loss from platinum nanoparticles in Figure 1, experimental data and reference spectrum are plotted. B. X-ray energy-dispersive spectrum taken from aluminum needle; inset shows normalized spectrum.

TABLE 1. Summary of aluminum etching conditions and corresponding platinum deposition.

\begin{tabular}{|c|c|}
\hline Etching Conditions & Platinum Present \\
\hline AC $(15-20 \mathrm{~V})$ & Yes \\
\hline Pulsed DC $(1 / 2$ wave rectified AC) & No \\
\hline AC followed by Pulsed DC & No \\
\hline
\end{tabular}

